\title{
Schizophrenia, Carbonyl Stress and Carnosine
}

\author{
Alan R Hipkiss* \\ Aston Research Centre for Healthy Ageing (ARCHA), Aston University, United Kingdom \\ *Corresponding author: Alan R Hipkiss, Aston Research Centre for Healthy Ageing (ARCHA), Aston University, Birmingham, United \\ Kingdom
}

\begin{abstract}
Recent research suggests that schizophrenia is associated with the development of an advanced aging phenotype (carbonyl stress) and erythrocytes from schizophrenics also exhibit symptoms of cellular aging (increased levels of glycated proteins and ubiquitinated proteins), possibly due to excessive glycolysis-induced methylglyoxal (MG) generation. The endogenous dipeptide carnosine (beta-alanyl-L-histidine), which can delay cellular aging, suppress glycolysis and inhibit MG-induced protein glycation, also exerts some beneficial effects towards schizophrenia. Carnosine is present in human erythrocytes and the olfactory bulb (olfactory dysfunction is associated with schizophrenia). It is suggested that enhanced erythrocyte and olfactory carnosine levels may be more therapeutic towards schizophrenia, if carnosine was also administered intra-nasally to avoid serum carnosinase activity.
\end{abstract}

Keywords: Carnosine; glycation; methylglyoxal; erythrocyte; aging; nasal administration

\section{Introduction}

\section{Schizophrenia and carbonyl stress}

Many studies have indicated a relationship between schizophrenia and dysfunctional energy metabolism [1-3] whilst others indicate that carbonyl stress and generation of advanced glycation end-products (AGEs) accompany schizophrenia [4,5]. Furthermore, a recent study suggests that changes in glycolysis and accelerated cellular aging in glial cells contribute to the condition [6]. The glycolytic intermediates glyceraldehyde-3-phosphate and dihydroxyacetone-phosphate are the most likely sources of AGE formation due to their ability to spontaneously decompose into methylglyoxal (MG). MG is well recognized as a major glycating agent and is thought to be responsible for much macromolecular modifications associated with type- 2 diabetes and age-related neurodegenerative conditions $[7,8]$. However, there is no clear evidence whether suppression of MG generation, via decreased glycolytic activity, has any effect on schizophrenia. The suggestion that schizophrenia seems to be associated with accelerated cellular aging [6] is supported by another recent observation reporting that erythrocytes obtained from schizophrenics contain elevated mounts of ubiquitinated proteins [9]. This might arise from either increased generation of targets for ubiquitination (e.g. aberrant polypeptides or denatured misfolded proteins), or decreased de-ubiquitinating activity, or decreased proteasomal proteolytic activity which would normally complete polypeptide destruction. Interestingly, MG and other agents responsible for carbonyl stress, also induce protein cross-linking which not only renders the target protein less susceptible to proteolytic attack but can also result in inhibition of proteasome activity generally [10]. Thus, it is conceivable that excessive glycolysis can provoke an aging phenotype (AGE accumulation and proteostatic dysfunction) via increased MG generation; such a relationship has been demonstrated in mice fed a high glycemic- index diet [11]. Never-the-less it is necessary to show whether glycation compromises proteostatic in erythrocytes from schizophrenics.

\section{Erythrocytes and schizophrenia}

A number of recent papers have revealed that erythrocytes obtained from patients with neurological problems, such as Alzheimer's Disease (AD) and Parkinson's Disease (PD), exhibit symptoms typical of aging cells in general. For example, compromised proteolytic activity and MG detoxification were detected in AD erythrocytes [12] and accumulation of aggregated protein occurs in red cells from PD patients [13]. Furthermore, dysfunctional energy metabolism, especially in relation to glycolysis culminating in carbonyl stress, are now regarded as characteristics of both AD and PD $[14,15]$. Therefore, it is not surprising that evidence of carbonyl stress is also accompanied by enhanced protein glycation 
[16] and accumulation of ubiquitinated proteins [9] in erythrocytes (and possibly other cells) obtained from schizophrenic individuals [17]. Moreover, one of the glycated proteins from "schizophrenic" red cells has been identified as a selenium-binding protein (SBP1) [18]; dysfunctional selenium metabolism has long been regarded as an important contributor to schizophrenia $[19,20]$. Selenium plays an important role in Sulphur metabolism required for synthesis of antioxidant enzymes such as glutathione peroxidase [21]. Thus, one is beginning to understand the relationship between AGE generation, carbonyl and oxidative stress and the apparently disparate biochemical attributes to schizophrenia.

\section{Carnosine, carbonyl stress and schizophrenia}

That erythrocytes can contain elevated amounts of MG and glycated proteins suggests the possibility that such red cells could become systemic sources of MG and AGEs to the brain and other tissues, following MG-induced eryptosis [22]. Consequently, it is important to consider whether suppression of carbonyl stress, not only in erythrocytes but in astrocytes and glia, could possibly be a therapeutic strategy. The naturally occurring dipeptide carnosine (beta-alanyl-L-histidine) has been shown to suppress glycolysis in cultured cells [23,24], delay replicative senescence [25], stimulate proteolysis of long-lived proteins in late passage cells [26] and inhibit AGE formation [27]. Furthermore, there is one study showing that schizophrenics subjected to dietary supplementation with carnosine exhibited some beneficial effects [28], possibly due to the dipeptide's pluripotent properties [29]. It is also interesting to note that

a) Olfactory dysfunction is also associated with schizophrenia $[30,31]$ and

b) Carnosine is enriched in the olfactory bulb [32].

Thus, one has to consider whether raising olfactory carnosine levels could also be useful. However, all studies employing dietary carnosine supplementation are subject to the problem of the presence of serum carnosinase activity which would destroy the dipeptide [33]. There is an alternative route however, which is to use an intra-nasal approach. This could involve a nasal spray of a carnosine solution; another approach could involve use of carnosine powder. Indeed "snorting" carnosine could be far more useful than most white powders some people use, be it illegal drugs or "medicinal snuff "of old. In fact, intra-nasal delivery of potential therapeutic agents is currently being explored [34] with respect to neurodegenerative conditions, as proposed many years ago [35].

Carnosine has been detected in human erythrocytes [36] but in lower amounts when obtained from elderly individuals [36]. It is presumed that red cell carnosine is synthesized (from betaalanine and histidine) during erythropoiesis. Consequently, it would be useful to determine whether dietary supplementation with carnosine or beta-alanine raises erythrocyte carnosine levels and whether there are any beneficial effects with respect to the recognized changes in "schizophrenic" erythrocytes. Additionally, it is suggested that any carnosine (dietary or nasally administered) supplementation period should last for at least 120 days to ensure maximal numbers of carnosine-enriched erythrocytes. It has been proposed that excessive and continuous glycolysis in erythrocytes enhances red cell MG levels, and thus also facilitate delivery of erythrocyte MG to the tissues including the brain [22]. Consequently, it will be also important to determine whether such supplementation protocols decrease carbonyl stress and MG levels not only in red cells but the tissues generally including glia [6].

\section{References}

1. Iwata K (2019) Mitochondrial Involvement in Mental Disorders; Energy Metabolism, Genetic, and Environmental Factors. Methods Mol Biol 1916: $41-48$

2. Sullivan CR, Mielnik CA, O Donovan SM, Funk AJ, Bentea E, et al. (2019) Connectivity Analyses of Bioenergetic Changes in Schizophrenia: Identification of Novel Treatments. Mol Neurobiol 56(6): 4492-4517.

3. Duarte JMN, Xin L (2019) Magnetic Resonance Spectroscopy in Schizophrenia: Evidence for Glutamatergic Dysfunction and Impaired Energy Metabolism. Neurochem Res 44(1): 102-116.

4. Ohnuma T, Nishimon S, Takeda M, Sannohe T, Katsuta N, et al. (2018) Carbonyl Stress and Microinflammation-Related Molecules as Potential Biomarkers in Schizophrenia. Front Psychiatry 9: pp. 82.

5. Fu J, Zuo X, Yin J, Luo X, Li Z, et al. (2017) Association of Polymorphisms of the Receptor for Advanced Glycation Endproducts Gene with Schizophrenia in a Han Chinese Population. Biomed Res Int 6379639.

6. Zuccoli GS, Guest PC, Martins-de-Souza D (2019) Effects on Glial Cell Glycolysis in Schizophrenia: An Advanced Aging Phenotype? Adv Exp Med Biol 1178: 25-38.

7. Schalkwijk C, Stehouwer CD (2019) Methylglyoxal, a highly reactive dicarbonyl compound, in diabetes, its vascular complications and other age-related diseases. Physiol Rev 100(1): 407-461.

8. Vicente Miranda H, Szego ÉM, Oliveira LMA, Breda C, Darendelioglu E, et al. (2017) Glycation potentiates $\alpha$-synuclein-associated neurodegeneration in synucleinopathies. Brain 140(5): 1399-1419.

9. Bousman CA, Luza S, Mancuso SG, Kang D, Opazo CM, et al. (2019) Elevated ubiquitinated proteins in brain and blood of individuals with schizophrenia. Sci Rep 9(1): 2307.

10. Queisser MA, Yao D, Geisler S, Hammes HP, Lochnit G, et al. (2010) Hyperglycemia impairs proteasome function by methylglyoxal. Diabetes 59(3): 670-678.

11. Uchiki T, Weikel KA, Jiao W, Shang F, Caceres A, et al. (2012) Glycationaltered proteolysis as a pathobiologic mechanism that links dietary glycemic index, aging, and age-related disease (in nondiabetics). Aging Cell 11(1): 1-13.

12. Lv H, Wei GY, Guo CS, Deng YF, Jiang YM, et al. (2020) 20S proteasome and glyoxalase 1 activities decrease in erythrocytes derived from Alzheimer's disease patients. Neural Regen Res 15(1): 178-183.

13. Matsumoto J, Stewart T, Sheng L, Li N, Bullock K, etal. (2017) Transmission of $\alpha$-synuclein-containing erythrocyte-derived extracellular vesicles across the blood-brain barrier via adsorptive mediated transcytosis: another mechanism for initiation and progression of Parkinson's disease? Acta Neuropathol Commun 5(1): 1-71.

14. Butterfield DA, Halliwell B (2019) Oxidative stress, dysfunctional glucose metabolism and Alzheimer disease. Nat Rev Neurosci 20(3): 148-160.

15. Smith AM, Depp C, Ryan BJ, Johnston GI, Alegre Abarrategui J, et al. (2018) Mitochondrial dysfunction and increased glycolysis in prodromal and early Parkinson's blood cells. Mov Disord 33(10): 1580-1590.

16. Hagen JM, Sutterland AL, Koeter MW, Lutter R, Cohen D, et al. (2017) Advanced Glycation End Products in Recent-Onset Psychosis Indicate Early Onset of Cardiovascular Risk. J Clin Psychiatry 78(9): 1395-1401.

17. Olivero P, Lozano C, Sotomayor Zárate R, Meza-Concha N, Arancibia M, et al. (2018) Proteostasis and Mitochondrial Role on Psychiatric 
and Neurodegenerative Disorders: Current Perspectives. Neural Plast 6798712.

18. Ishida YI, Kayama T, Kibune Y, Nishimoto S, Koike S, et al. (2017) Identification of an argpyrimidine-modified protein in human red blood cells from schizophrenic patients: A possible biomarker for diseases involving carbonyl stress. Biochem Biophys Res Commun 493(1): 573577.

19. Amar S, Ovadia O, Maier W, Ebstein R, Belmaker RH, et al. (2010) Copy number variation of the SELENBP1 gene in schizophrenia. Behav Brain Funct 6: 40 .

20. Udawela M, Money TT, Neo J, Seo MS, Scarr E, et al. (2015) SELENBP1 expression in the prefrontal cortex of subjects with schizophrenia. Transl Psychiatry 5(8): e615.

21. Solovyev ND (2015) Importance of selenium and selenoprotein for brain function: From antioxidant protection to neuronal signalling. J Inorg Biochem 153: 1-12

22. Hipkiss AR (2019) The human erythrocyte can become both a metabolic "Achilles'Heel" and a "Trojan Horse": Likely consequences of persistent excessive glycolysis. Integr Food Nutr Metabolism 6: 1-2.

23. Shen Y, Yang J, Li J, Shi X, Ouyang L, et al. (2014) Carnosine inhibits the proliferation of human gastric cancer SGC-7901 cells through both of the mitochondrial respiration and glycolysis pathways. PLoS One 9(8): e104632.

24. Renner C, Asperger A, Seyffarth A, Meixensberger J, Gebhardt R, et al. (2010) Carnosine inhibits ATP production in cells from malignant glioma. Neurol Res 32(1): 101-105.

25. McFarland GA, Holliday R (1999) Further evidence for the rejuvenating effects of the dipeptide L-carnosine on cultured human diploid fibroblasts. Exp Gerontol 34(1): 35-45.

26. Hipkiss AR, Brownson C, Bertani MF, Ruiz E, Ferro A (2002) Reaction of carnosine with aged proteins: another protective process? Ann N Y Acad Sci 959: 285-294
27. Ghodsi R, Kheirouri S (2018) Carnosine and advanced glycation end products: A systematic review. Amino Acids 50(9): 1177-1186.

28. Chengappa KN, Turkin SR, De Santi S, Bowie CR, Brar JS, et al. (2012) A preliminary, randomized, double-blind, placebo-controlled trial of L-carnosine to improve cognition in schizophrenia. Schizophr Res 142(1-3): 145-152.

29. Artioli GG, Sale C, Jones RL (2019) Carnosine in health and disease. Eur J Sport Sci 19(1): 30-39.

30. Kamath V, Crawford J, DuBois S, Nucifora FC, Nestadt G, et al. (2019) Contributions of olfactory and neuropsychological assessment to the diagnosis of first-episode schizophrenia. Neuropsychology 33(2): 203211.

31. Kamath V, Lasutschinkow P, Ishizuka K, Sawa A (2018) Olfactory Functioning in First-Episode Psychosis. Schizophr Bull 44(3): 672-680.

32. Khama Murad AX, Pavlinova LI, Mokrushin AA (2008) Neurotropic effect of exogenous L-carnosine in cultured slices of the olfactory cortex from rat brain. Bull Exp Biol Med 146(1): 1-3.

33. Zhang S, Albrecht T, Rodriguez Niño A, Qiu J, Schnuelle P, et al. (2019) Carnosinase concentration, activity, and CNDP1 genotype in patients with type 2 diabetes with and without nephropathy. Amino Acids 51(4): 611-617.

34. Bermúdez ML, Skelton MR, Genter MB (2018) Intranasal carnosine attenuates transcriptomic alterations and improves mitochondrial function in the Thy1-aSyn mouse model of Parkinson's disease. Mol Genet Metab 125(3): 305-313.

35. Hipkiss AR (2005) Could carnosine suppress zinc-mediated proteasome inhibition and neurodegeneration? Therapeutic potential of a non-toxic but non-patentable dipeptide. Biogerontol 6(2): 147-149.

36. Chaleckis R, Murakami I, Takada J, Kondoh H, Yanagida M (2016) Individual variability in human blood metabolites identifies age-related differences. Proc Natl Acad Sci USA 13(16): 4252-4259.

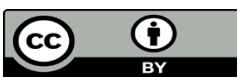

This work is licensed under Creative Commons Attribution 4.0 License

To Submit Your Article Click Here: Submit Article

DOI: $10.32474 /$ JJNBD.2019.03.000168

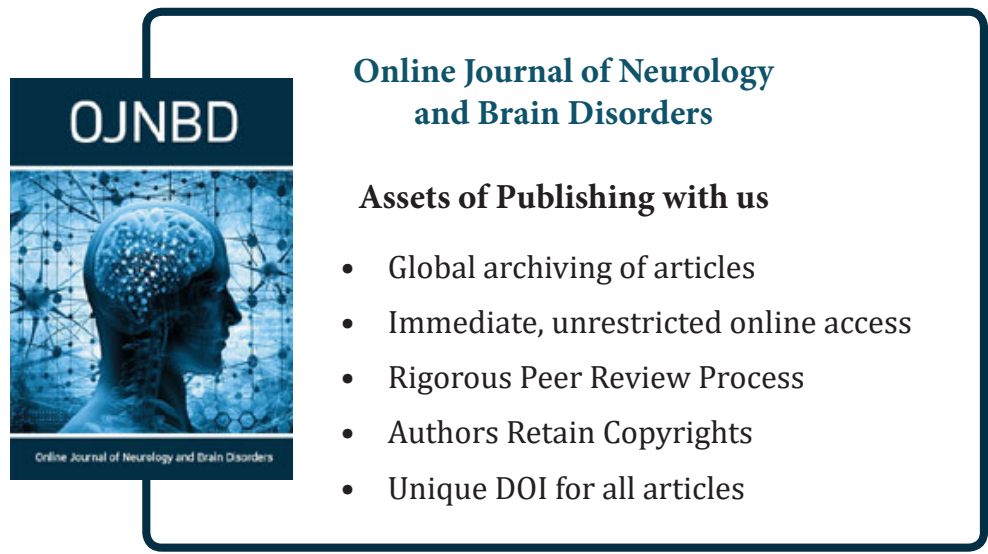

